\title{
Mean T Wave Area
}

National Cancer Institute

\section{Source}

National Cancer Institute. Mean T Wave Area. NCI Thesaurus. Code C62148.

The average (mean) "area under the curve" of the deflection from baseline of the T wave (representing ventricular repolarization), obtained from a set of measurements of the T wave, from a single lead or set of leads. 